\title{
The influence of graphene nano-particles on the biological interest of polyvinyl alcohol nanocomposites
}

\begin{abstract}
Recently, the development of Nano-material has become the most interest issues for many researchers. For example, nanocarbons such as graphene $(\mathrm{Gr})$ and graphene derivatives attracted more attention due to their better structural, high strength, low density, and good combability with polymer. Introduction these nanocarbon to the polymer matrix like polyvinyl alcohol (PVA) improved the properties of the polymer for instance: thermal, mechanical, and electrical properties. This led to its accessibility to many applications. This review, present recent studies of preparation PVA nanocomposites with $\mathrm{Gr}$ as filler and also illiterate the improvement of properties for further use.
\end{abstract}

Keywords: nano-material, polyvinyl alcohol, graphene, polymer nanocomposite.
Volume 3 Issue I - 2017

\author{
Khadejah S Hajeeassa,' Mahmoud A \\ Hussein, ${ }^{1,2}$ Zahra MAl-amshany' \\ 'Chemistry Department, King Abdulaziz University, Saudi Arabia \\ ${ }^{2}$ Chemistry Department, Polymer Chemistry Lab I22, Egypt
}

\author{
Correspondence: Mahmoud A Hussein, Chemistry \\ Department, Faculty of Science, King Abdulaziz University, PO \\ Box 80203, Jeddah 21589, Saudi Arabia, \\ Email mahussein74@yahoo.com; maabdo@kau.edu.sa, \\ mahmali@aun.edu.eg
}

Received: July 28, 2017 | Published: September 07, 2017

\section{Introduction}

Nanocomposite is a multiphase material that made from a combination of two or more of constituent material (matrix and reinforcing phase) with different physical or chemical properties that, when they combined, the resulting material has characteristics differently from the individual compounds. ${ }^{1}$ Over the past decades, nanocomposites become a magnificent issue in our lives. Materials play the key role in every area of technology of the $21^{\text {st }}$ century. It started to be used in medicals, industrials, computing, and sensor applications to make our lives more comfortable.,3 One kind of nanocomposites according to the matrix is polymer nanocomposite. Polymer nanocomposites are a combination of polymer or copolymer and Nano-sized fillers (less than $5 \mathrm{wt} \%$ ). Addition of nanofiller to the polymer matrix improve properties and can use in different application. ${ }^{4}$

Polyvinyl alcohol (PVA) is the most important polymer which is highly biocompatible, highly water-soluble, nontoxic, and synthetic polymer. PVA, in addition has been used widely in biological applications and also sensitive for water and humidity. ${ }^{5}$ PVA can be used as a matrix for fabricate polymer nanocomposites and use it in huge application. ${ }^{6}$ Nanocarbons are considered as carbon-based materials which can be bonded at the molecular level by different ways to improve unique properties. ${ }^{7}$ Carbon nanofillers like graphene and carbon nanotube plays an important role because of their great structure and efficacious properties e.g. thermal, electrical, and mechanical properties etc. ${ }^{89}$ Graphene (Gr) is a single layer twodimensional (2D) carbon atoms arranged in a honeycomb pattern, has been invited particularly by technologists and chemists' researchers due to unique properties like thermal, electrical, and mechanical properties. ${ }^{10,11}$. In this review, we focus and highlight the enhancement of properties and show some application of PVA with graphene as filler.

\section{Graphene nanoparticles (GNP)}

Carbon has been known to form many allotropes because of its ability to exist in different hybridizations. Different allotropes of carbon exist in nature such as graphite, diamond, fullerenes and amorphous carbon. Figure 1 shows the structures of some of the carbonaceous materials existing in nature. ${ }^{12}$ Among these, graphene is the building block of carbonaceous materials existing in nature with unique properties principally attributed to carbon atoms arranged into two-dimensional (2D) planer monolayer of sp2-hybridized orbitals with honeycomb (hexagonal) lattice structure. ${ }^{13}$

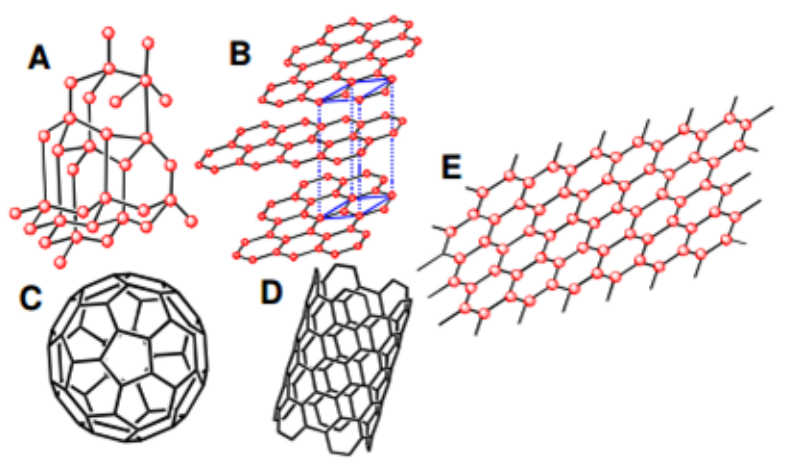

Figure I The structures of (A) diamond (B) graphite (stacked layers of many graphene sheets bonded together by weak van derWaals forces). (C) fullerene (C60) (D) SWCNTs and (E) GNP.12,14

\section{Properties of graphene nanoparticles}

Graphene Nanoparticles exhibits a high level of electrical conductivity $\left(7200 \mathrm{Sm}^{-1}\right)$ due to the unique nanostructures; also the electron and hole in graphene sheets have extremely high carrier mobility behave like massless relativistic particles at room temperature $\left(10,000-15,000 \mathrm{~cm}^{2} \mathrm{~V}^{-1} \mathrm{~S}^{-1}\right)$. Pristine graphene is highly thermally conductive $\left(\sim 5000 \mathrm{~W} \mathrm{~m}^{-1} \mathrm{~K}^{-1}\right)$, with superior mechanical properties with a high Young's modulus $(\sim 1.0 \mathrm{TPa})$, these properties along with the large specific area $\left(2630 \mathrm{~m}^{2} \mathrm{~g}^{-1}\right){ }^{15,16}$ Two carbon atoms are bonded together with a $\sigma \mathrm{C}-\mathrm{C}$ chemical bond which is an extremely strong bond in materials due to hybridized orbitals 
generated by the superposition of $2 \mathrm{~s}$ with $2 \mathrm{p}_{\mathrm{x}}$ and $2 \mathrm{p}_{\mathrm{y}}$ orbitals. The planar orbitals form the energetically stable and localized $\sigma$-bonds with the three nearest-neighbor carbon atoms in the honeycomb lattice, and they are responsible for most of the binding energy and for the elastic properties of the graphene sheet. The remaining free $2 p_{z}$ orbitals perpendicularly oriented to the molecular plane hybridizes to form the conduction $(\pi)$ and valence $\left(\pi^{*}\right)$ bands, which is responsible of the electron conduction of graphene ${ }^{15,17}$ pure graphene sheets are highly hydrophobic(non-dispersible in aqueous solution) in nature and easily agglomerates because of the strong $\pi-\pi$ interactions along high surface area of graphene. ${ }^{12,18}$

\section{Polyvinyl alcohol/graphene nanocomposites}

Nanocomposite based of PVA and Gr have been synthesized and analyzed by many researchers to improve material's properties. The strong interaction between PVA and Gr and because of this it led to use in different application. ${ }^{19}$ As an example of PVA with Gr as filler, Rade S et al. 2015, prepared PVA/Gr by simple dissolution technique. The introduction of Gr to PVA improves mechanical and thermal properties

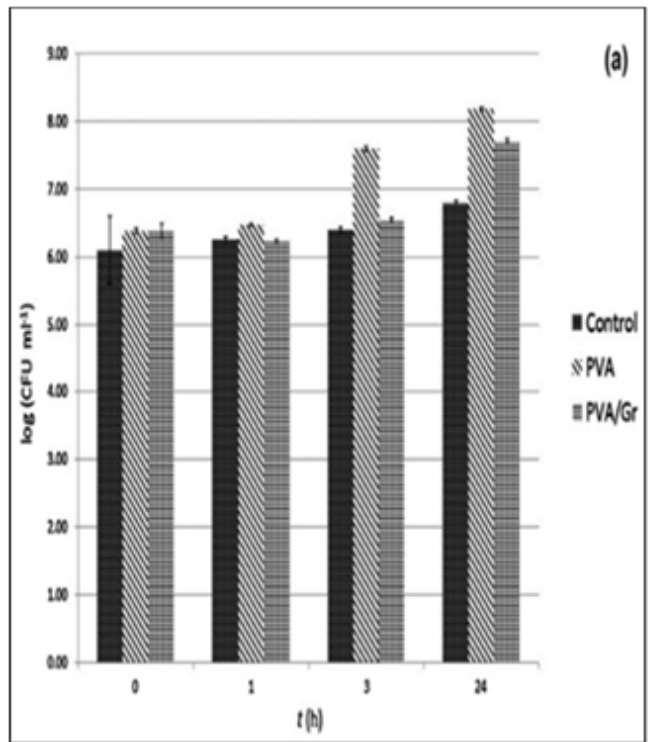

comparing with neat PVA. In this study, PVA/Gr nanocomposite was nontoxic for peripheral bloods nanomolecular cells (PBMC) and exhibited a strong antibacterial activity against $E$. coli (gram negative bacteria) and $S$. aureus (gram positive bacteria) that made it as an excellent candidate biomedical application (6) presented in Figure 2. Other study by Yuang-Cheng $\mathrm{C}$ et al., ${ }^{20}$ also showed the different addition of $\mathrm{Gr}$ from 1\%-10\% improved the antibacterial activity against $E$. coli by $92 \%-97.1 \%$ and $92.3 \%-99.7 \%$ against $S$. aureus. ${ }^{20}$ In order to improve the biological application, PVA/Gr/AgNPs nanocomposites hydrogel synthesized by Abudabbus $\mathrm{M}$ et al., ${ }^{21}$ silver nanoparticles (AgNPs) in this study used as agent for dressing antimicrobial material. The results showed the lowest cytotoxicity to PBMC culture and good inhibition for bacteria. ${ }^{21}$ Moreover, different types of nanofiller also cooperated with PVA. Recent study by Adel U et al., ${ }^{22} \mathrm{PVA} / \mathrm{graphene}$ oxide/starch/silver in form (PVA/GO/starch/ $\mathrm{Ag}$ ) nanocomposites prepared by simple solvent method. The dimeter of zoon inhibition increased for the prepared nanocomposite against E. coli $17 \mathrm{~mm}$ and S. aureus $20.5 \mathrm{~mm}$ as illustrated in Figure 3, while pure PVA did not show any antibacterial response. ${ }^{22}$

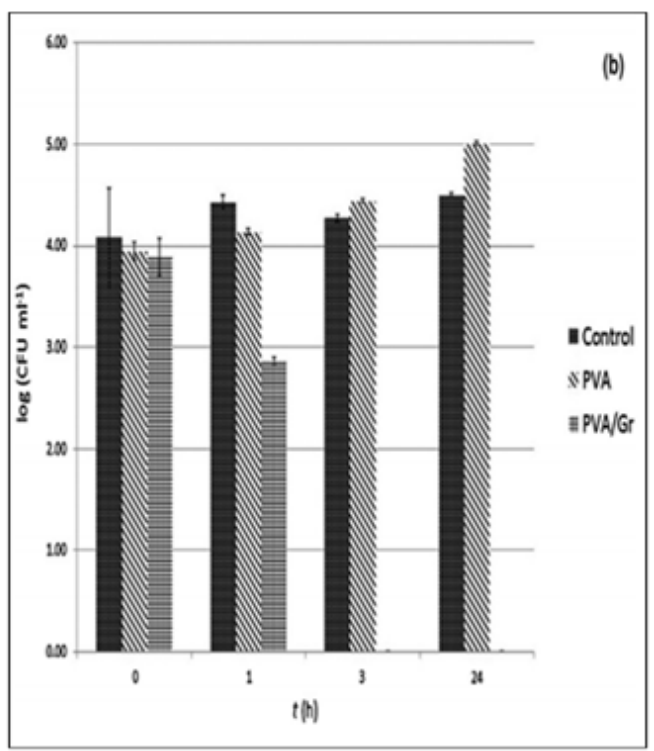

Figure 2 Viable cell number of (A) E. coli and (B) S. aureus after contact with PVA and PVA/Gr hydrogels for 0, I, 3, and $24 \mathrm{~h}$ in PB as compared to the control w/o samples.

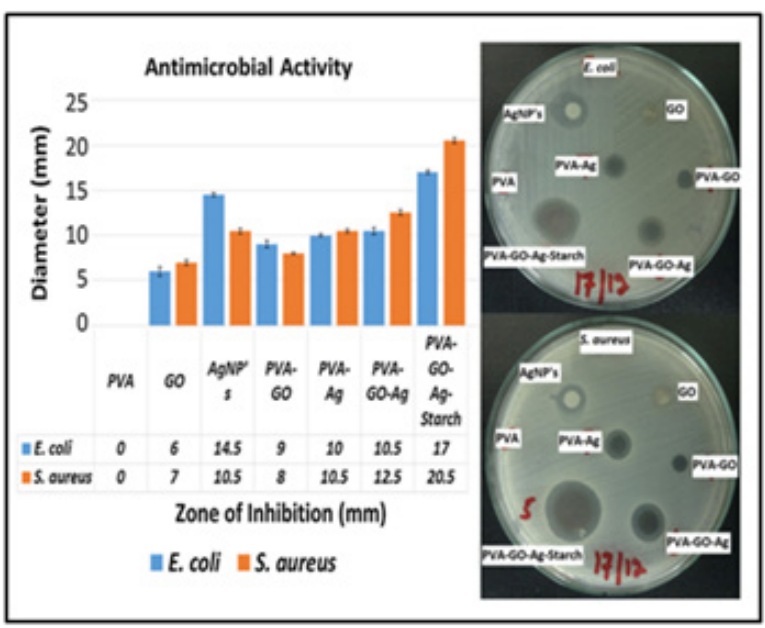

Figure 3 Statistical representation of antimicrobial activity of polymer nanocomposites films against $E$. coli and S. aureus.

\section{Conclusion}

One of the magnificent accomplishment in nanoscience and nanotechnology is polymer nanocomposites. The development of polymer nanocomposites has massive impact on biological research recently. This review considers brief summary the recent studies of PVA/Graphene nanocomposites. The study also demonstrates the improvement effect on some types of bacteria.

\section{Acknowledgements}

None.

\section{Conflict of interest}

The author declares no conflict of interest.

\section{References}

1. Zhang J. Polymer nanocomposites: synthetic and natural fillers a review. Maderas: Ciencia y Tecnología. 2005;7(3):159-178. 
2. Kurahatti R, Surendranathan A, Kori S, et al. Defence applications of polymer nanocomposites. Defence Science Journal. 2010;60(5):551563.

3. Mandal A, Chakrabarty D. Studies on the mechanical, thermal, morphological and barrier properties of nanocomposites based on poly (vinyl alcohol) and nanocellulose from sugarcane bagasse. Journal of Industrial and Engineering Chemistry. 2014;20(2):462-473.

4. Denault J, Labrecque B. Technology group on polymer nanocompositesPNC-Tech. Canada: Industrial materials institute national research council Canada; 2004. 75 p.

5. Georgieva N, Bryaskova R, Tzoneva R. New Polyvinyl alcoholbased hybrid materials for biomedical application. Materials Letters. 2012;88:19-22.

6. Surudžić R, Janković A, Mitrić M, et al. The effect of graphene loading on mechanical, thermal and biological properties of poly (vinyl alcohol)/ graphene nanocomposites. Journal of Industrial and Engineering Chemistry. 2016;34:250-257.

7. Baughman RH, Zakhidov AA, De Heer WA. Carbon nanotubes-the route toward applications. Science. 2002;297(5582):787-792.

8. Balandin AA, Ghosh S, Bao W, et al. Superior thermal conductivity of single-layer graphene. Nano letters. 2008;8(3):902-907.

9. Lee C, Wei X, Kysar JW, et al. Measurement of the elastic properties and intrinsic strength of monolayer graphene. Science. 2008;321(5887):385388.

10. Cai YM, Qin ZY, Long C. Effect of electrolytes on electrochemical properties of graphene sheet covered with polypyrrole thin layer. Progress in Natural Science: Materials International. 2011;21(6):460-466.

11. Wang L, Wu Y, Chen F, et al. Photocatalytic enhancement of Mg-doped $\mathrm{ZnO}$ nanocrystals hybridized with reduced graphene oxide sheets. Progress in Natural Science: Materials International. 2014;24(1):6-12.

12. Potts JR, Dreyer DR, Bielawski CW. Ruoff, R. S. Graphene-based polymer nanocomposites. Polymer. 2011;52(1):5-25.
13. Pumera M, Ambrosi A, Bonanni A, et al. Graphene for electrochemical sensing and biosensing, TrAC Trends in Analytical Chemistry. 2010; 9(9):954-965.

14. Choi W, Lee LW. Graphene: synthesis and applications. USA: CRC Press; 2011. 394 p.

15. Kuilla T, Bhadra S, Yao D, et al. Recent advances in graphene based polymer composites. Progress in Polymer Science. 2010;35(11):1350 1375.

16. Inagaki M, Kang F. Graphene derivatives: graphane, fluorographene, graphene oxide, graphyne and graphdiyne. Journal of Materials Chemistry A. 2014;2:13193-13206.

17. Chee W, Lim H, Huang N, Harrison I. Nanocomposites of graphene/ polymers: a review. RSC Advances. 2015;5:68014-68051.

18. Viculis LM, Mack JJ, Mayer OM, et al. Intercalation and exfoliation routes to graphite nanoplatelets. Journal of Materials Chemistry. 2005;15:974-978.

19. Zhang C, Liu T. A review on hybridization modification of graphene and its polymer nanocomposites. Chinese science bulletin. 2012;57(23):3010 3021.

20. Cao YC, Wei W, Liu J, et al. The preparation of graphene reinforced poly (vinyl alcohol) antibacterial nanocomposite thin film. International Journal of Polymer Science. 2015. 7 p.

21. Abudabbus M, Jevremović I, Janković A, et al. Biological activity of electrochemically synthesized silver doped polyvinyl alcohol/graphene composite hydrogel discs for biomedical applications. Composites Part B: Engineering. 2016;104:26-34.

22. Usman A, Hussain Z, Riaz A, et al. Enhanced mechanical, thermal and antimicrobial properties of poly (vinyl alcohol)/graphene oxide/starch/ silver nanocomposites films. Carbohydr polym. 2016;153:592-599. 